\title{
Avaliação dos Efeitos Sobre a Qualidade de Vida da Mulher Pós-menopáusica Histerectomizada com o Uso de Implantes Subcutâneos Biodeğradáveis de 17-beta-estradiol
}

Autor: João Sabino de Lima Pinho Neto

Tese de Livre-Docência em Ginecologia apresentada ao Departamento de Ginecologia, Obstetrícia e Reprodução Humana da Faculdade de Medicina da Universidade Federal da Bahia, em 15 de dezembro do 2002.

Os efeitos dos implantes subcutâneos biodegradáveis de 17-beta-estradiol sobre a qualidade de vida de mulheres, histerectomizadas, na pós-menopausa, foram avaliados em estudo clínico ao longo de seis meses. Foram incluídas neste estudo 83 pacientes, das quais 60 concluíram o estudo. A qualidade de vida foi avaliada de acordo com os resultados de uma bateria de questionários padronizados. A qualidade de vida melhorou significativamente com o uso dos implantes de estradiol quando comparada com os resultados pré-tratamento, principalmente no que concerne aos aspectos da qualidade de vida relacionados à saúde ( $\mathrm{p}<0,001)$ e ao bem- estar ( $p<0,001)$, aos sintomas específicos do climatério, incluindo problemas ( $\mathrm{p}<0,001)$ e disfunções sexuais (p $<0,01)$ e ao alívio dos sintomas estimados pelas próprias pacientes ( $\mathrm{p}<0,0001)$.

Conclui-se que as pacientes que fazem uso de implantes subcutâneos biodegradáveis de 17-beta-estradiol, durante seis meses, apresentam importante alívio dos sintomas climatéricos e grande melhora na qualidade de vida.

Palavras-chave: Menopausa. Terapia de reposição hormonal.

\section{Diağnóstico da Restrição de Crescimento Fetal pela Relação Diâmetro Transverso do Cerebelo/Circunferência Abdominal}

Autor: José de Arimatea Barreto

Orientador: Prof. Dr. Carlos Augusto Alencar Júnior

Tese apresentada ao Curso de Mestrado em Tocoginecologia do Departamento de Saúde Materno-Infantil da Universidade Federal do Ceará em 21/05/2003.

Correspondência:

José de Arimatea Barreto

Rua Álvaro Fernandes, 44 - Bairro Damas

60420-570 - Fortaleza - Ceará

Fone: (085)-2923119 / (085)-99813908 / Fax: (085)-2816000

e-mail: arimateabarreto@uol.com.br

Objetivo: testar a validade da relação diâmetro transverso do cerebelo (DTC) / circunferência abdominal (CA) no diagnóstico da restrição de crescimento fetal (RCF), determinando seu melhor ponto de corte, sua acurácia nas restrições simétrica e assimétrica, além de compará-la com a relação comprimento do fêmur (CF)/ circunferência abdominal (CA).

Métodos: estudo de teste diagnóstico prospectivo, transversal, envolvendo 250 gestantes. Os neonatos cuja relação DTC/CA foi maior do que o do ponto de corte foram considerados acometidos por RCF. Idem para a relação CF/CA. Considerou-se padrão-ouro para o diagnóstico de RCF recém-nascidos cujos pesos situaram-se abaixo do percentil 10 para a idade gestacional nas curvas de Lubchenco et al. (1963).

Resultados: O ponto de corte da relação DTC/CA, obtido através de curva ROC ( receiver operator characteristic), foi 16,1. A sensibilidade, especificidade, valores preditivos positivo e negativo, razões de verossimilhan- ca positiva e negativa foram de $77,4 \%, 82,6 \%, 38,7 \%$, $96,3 \%, 4,5$ e 3,7, respectivamente. Na RCF simétrica a sensibilidade e especificidade foram de $80,8 \%$ e $81,7 \%$, respectivamente. $\mathrm{Na}$ assimétrica a sensibilidade e especificidade foram $60 \%$ e $75 \%$, respectivamente. Os resultados foram menores do que os observados na simétrica, porém, não estatisticamente significantes ( $p>0,05)$. O ponto de corte da relação $\mathrm{CF} / \mathrm{CA}$ foi de 22,6 , com sensibilidade, especificidade, valores preditivos positivo e negativo, razões de verossimilhança positiva e negativa de $67,7 \%, 81,7 \%, 34,4 \%, 94,7 \%, 3,7$ e 2,5 , respectivamente.

Conclusões: a relação DTC/CA mostrou-se eficaz no diagnóstico da $\mathrm{RCF}$, tanto simétrica quanto assimétrica. $A$ relação $\mathrm{CF} / \mathrm{CA}$ mostrou-se menos eficaz.

Palavras-chave: Restrição de crescimento fetal. Diâmetro cerebelar transverso. 